\title{
Multivariate extensions of the Golden-Thompson inequality
}

\author{
Frank Hansen
}

June 22, 2014

\begin{abstract}
We study concave trace functions of several operator variables and formulate and prove multivariate generalisations of the GoldenThompson inequality. The obtained results imply that certain functionals in quantum statistical mechanics have bounds of the same form as they appear in classical physics.
\end{abstract}

MSC2010 classification: 47A63.

Key words and phrases: Golden Thompson's inequality; multivariate trace inequality; concave trace function.

\section{Introduction}

The Golden-Thompson inequality, which is of importance in statistical mechanics and in the theory of random matrices, states that

$$
\operatorname{Tr} e^{L+B} \leq \operatorname{Tr} e^{L} e^{B}
$$

for arbitrary self-adjoint matrices $L$ and $B$. It is known that there is no direct extension of this inequality to more operator variables, and there is an extensive literature investigating these matters, cf. [6, 1, 3] and the references therein.

We prove, among other statements, the following extension of the GoldenThompson inequality. Consider $n \times m$ matrices $H_{1}, \ldots, H_{k}$ with

$$
H_{1}^{*} H_{1}+\cdots+H_{k}^{*} H_{k}=1_{n} .
$$


Then the inequality

$$
\operatorname{Tr} \exp \left(L+\sum_{i=1}^{k} H_{i}^{*} B_{i} H_{i}\right) \leq \operatorname{Tr} \exp (L) \sum_{i=1}^{k} H_{i}^{*}\left(\exp B_{i}\right) H_{i}
$$

is valid for arbitrary self-adjoint $n \times n$ matrices $L$ and $B_{1}, \ldots, B_{k}$. This is, for $n=m$, the same bound as obtained when all the matrices commute. We are thus allowed to estimate partition functions or the Helmhotz function in quantum statistical mechanics and obtain bounds on the same form as they appear in classical physics.

We obtain, as a simple special case, the inequality

$$
\operatorname{Tr} \exp \left(L+\frac{1}{2} A+\frac{1}{2} B\right) \leq \operatorname{Tr}(\exp L)\left(\frac{1}{2} \exp A+\frac{1}{2} \exp B\right)
$$

valid for arbitrary self-adjoint matrices $L, A$ and $B$. Notice that (2) reduces to the Golden Thompson inequality for $A=B$ and to convexity under the trace of the exponential function for $L=0$. The inequality may thus be considered as an interpolation inequality between Golden-Thompson's inequality and Jensen's inequality. However, we cannot derive (2) from these special cases. If we first apply Golden-Thompson's inequality then we obtain

$$
\operatorname{Tr} \exp \left(L+\frac{1}{2} A+\frac{1}{2} B\right) \leq \operatorname{Tr}(\exp L) \exp \left(\frac{1}{2} A+\frac{1}{2} B\right)
$$

but this is insufficient to obtain (2) since $L$ is arbitrary and the exponential function is not operator convex.

\section{Preliminaries}

The following lemma is both well-known and very useful. We include the proof for the benefit of the reader.

Lemma 2.1. Let $\varphi: \mathcal{D} \rightarrow \mathcal{A}_{\text {sa }}$ be a map defined in a convex cone $\mathcal{D} \subseteq X$ of a Banach space $X$ with values in the self-adjoint part of a $C^{*}$-algebra $\mathcal{A}$. If $\varphi$ is Fréchet differentiable, convex and positively homogeneous then

$$
d \varphi(x) h \leq \varphi(h) \quad x, h \in \mathcal{D}
$$

where $d \varphi(x)$ denotes the Fréchet differential of $\varphi(x)$. 
Proof. Since

$$
\begin{aligned}
\varphi(x+t h) & =(1+t) \varphi\left(\frac{1}{1+t} x+\frac{t}{1+t} h\right) \\
& \leq(1+t)\left(\frac{1}{1+t} \varphi(x)+\frac{t}{1+t} \varphi(h)\right) \\
& =\varphi(x)+t \varphi(h)
\end{aligned}
$$

for $0 \leq t \leq 1$, we obtain

$$
\frac{\varphi(x+t h)-\varphi(x)}{t} \leq \varphi(h) \quad 0<t \leq 1
$$

and thus $d \varphi(x) h \leq \varphi(h)$. QED

We refer to the monograph [2] for a general account of Fréchet differentiable mappings between Banach spaces.

The logarithm is operator monotone with Lebesgue measure as representing measure, thus

$$
\log x=\int_{0}^{\infty}\left(\frac{1}{t+1}-\frac{1}{x+t}\right) d t \quad x>0 .
$$

Since

$$
x^{1 / 2}(x+h)^{-1} x^{1 / 2}=\left(1+x^{-1 / 2} h x^{-1 / 2}\right)^{-1}=1-x^{-1 / 2} h x^{-1 / 2}+o(h),
$$

we derive that $d x^{-1} h=-x^{-1} h x^{-1}$. Notice that $h$ may be arbitrary as we are not using the functional calculus. Consequently

$$
d \log (x) h=\int_{0}^{\infty}(x+t)^{-1} h(x+t)^{-1} d t .
$$

We have thus obtained the following integral expression

$$
Q(x, h)=\operatorname{Tr} h^{*} d \log (x) h=\operatorname{Tr} \int_{0}^{\infty} h^{*}(x+t)^{-1} h(x+t)^{-1} d t .
$$

It follows from the integral expression that $Q(x, h)$ is positively homogeneous in $(x, h)$. Lieb proved that it is a convex function in two variables [7, Theorem 3]. But this is a reflection of a quite general result. Zhang and the author 
recently proved [5] that for a strictly increasing continuously differentiable function $f:(0, \infty) \rightarrow \mathbf{R}$ the form

$$
(x, h) \rightarrow \operatorname{Tr} h^{*} d f(x) h \quad x>0
$$

is convex if and only if the derivative of $f$ is operator convex and numerically decreasing.

We shall now use Lemma 2.1. Notice that $Q(x, h)$ is defined in the cone $\mathcal{D}=B(H)_{+} \times B(H)$, where $H$ is a finite dimensional Hilbert space. Thus

$$
d Q(x, h)(y, k) \leq Q(y, k)
$$

for positive definite $x, y$ and arbitrary $h, k$.

We end this section by giving a new result for the form $Q$ that will prove crucial in the rest of the paper.

Proposition 2.2. Let $X$ be an invertible contraction. Then

$$
Q\left(X A X^{*}, B\right) \leq Q\left(A, X^{-1} B\left(X^{*}\right)^{-1}\right)
$$

for positive definite $A$ and arbitrary $B$.

Proof. We use the integral representation of the form $Q$ and obtain

$$
Q\left(X A X^{*}, B\right)=\operatorname{Tr} \int_{0}^{\infty} B^{*}\left(X A X^{*}+t\right)^{-1} B\left(X A X^{*}+t\right)^{-1} d t .
$$

Since $X$ is a contraction we derive the inequality

$$
\frac{1}{X A X^{*}+t} \leq \frac{1}{X(A+t) X^{*}}=\left(X^{*}\right)^{-1}(A+t)^{-1} X^{-1} .
$$

Under the trace this inequality implies

$$
\begin{aligned}
Q\left(X A X^{*}, B\right) & \leq \operatorname{Tr} \int_{0}^{\infty} B^{*}\left(X^{*}\right)^{-1}(A+t)^{-1} X^{-1} B\left(X^{*}\right)^{-1}(A+t)^{-1} X^{-1} d t \\
& =Q\left(A, X^{-1} B\left(X^{*}\right)^{-1}\right)
\end{aligned}
$$

which is the desired result. QED 


\section{Concave trace functions}

Theorem 3.1. Let $H$ be a contraction. Then the trace function

$$
\varphi(A)=\operatorname{Tr} \exp \left(H^{*}(\log A) H\right)
$$

is concave in positive definite matrices.

Proof. We may without loss of generality assume that $H$ is invertible. We calculate the first Fréchet differential

$$
\begin{aligned}
d \varphi(A) B & =\operatorname{Tr} d \exp \left(H^{*}(\log A) H\right)\left(H^{*}(d \log (A) B) H\right) \\
& =\operatorname{Tr} \exp \left(H^{*}(\log A) H\right)\left(H^{*}(d \log (A) B) H\right),
\end{aligned}
$$

where we used the identity $\operatorname{Tr} d f(A) B=\operatorname{Tr} f^{\prime}(A) B$ valid for differentiable functions. We then consider the following functions of the single operator variable $A$.

$$
\begin{aligned}
C & =H^{*}(\log A) H \\
D & =H^{*}(d \log (A) B) H=d_{A}\left(H^{*}(\log A) H\right) B=d_{A}(C) B \\
E & =H \exp (C) H^{*}=H \exp \left(H^{*}(\log A) H\right) H^{*} \\
G & =H d \exp (C)(D) H^{*}=d_{C}\left(H \exp (C) H^{*}\right) D=d_{C}(E) D .
\end{aligned}
$$

For clarity, we use the notation $d_{A}$ to indicate Fréchet differentiation with respect to $A$ of compound expressions. We proceed to calculate the second Fréchet differential

$$
\begin{aligned}
& d^{2} \varphi(A)(B, B)=d_{A}(d \varphi(A) B) B=d_{A}(\operatorname{Tr} \exp (C) D) B \\
& =\operatorname{Tr} d \exp (C)(D) D+\operatorname{Tr} \exp (C) H^{*} d^{2} \log (A)(B, B) H \\
& =\operatorname{Tr} d \exp (C)(D) D+\operatorname{Tr} E d^{2} \log (A)(B, B) .
\end{aligned}
$$

We recall [2] that $\varphi$ is concave if and only if $d^{2} \varphi(A)(B, B) \leq 0$ for positive definite $A$ and self-adjoint $B$. To evaluate the second term we apply the chain rule to the form $Q(A, B)$ and obtain

$$
\begin{aligned}
d Q(A, B)(a, b) & =d_{1} Q(A, B) a+d_{2} Q(A, B) b \\
& =\operatorname{Tr} B d^{2} \log (A)(B, a)+\operatorname{Tr} b d \log (A) B+\operatorname{Tr} B d \log (A) b
\end{aligned}
$$


for positive definite $A, a$ and self-adjoint $B, b$. The integral representation in (3) implies $\operatorname{Tr} B d \log (A) b=\operatorname{Tr} b d \log (A) B$, and we therefore obtain

$$
d Q(A, B)(a, b)=\operatorname{Tr} B d^{2} \log (A)(B, a)+2 \operatorname{Tr} b d \log (A) B .
$$

By using (4) we now obtain the inequality

$$
\begin{aligned}
\operatorname{Tr} a d^{2} \log (A)(B, B) & =\operatorname{Tr} B d^{2} \log (A)(B, a) \\
& =d Q(A, B)(a, b)-2 \operatorname{Tr} b d \log (A) B \\
& \leq Q(a, b)-2 \operatorname{Tr} b d \log (A) B
\end{aligned}
$$

for positive definite $A, a$ and self-adjoint $B, b$. Since $E$ is positive definite we may put $a=E$ and thus obtain

$$
\begin{aligned}
d^{2} \varphi(A)(B, B) & =\operatorname{Tr} d \exp (C)(D) D+\operatorname{Tr} E d^{2} \log (A)(B, B) \\
& \leq \operatorname{Tr} d \exp (C)(D) D+Q(E, b)-2 \operatorname{Tr} b d \log (A) B
\end{aligned}
$$

By setting $b=G$ we then obtain

$$
d^{2} \varphi(A)(B, B) \leq \operatorname{Tr} d \exp (C)(D) D+Q(E, G)-2 \operatorname{Tr} G d \log (A) B
$$

for positive definite $A$ and self-adjoint $B$. But since

$$
\begin{aligned}
\operatorname{Tr} G d \log (A) B & =\operatorname{Tr} H d \exp (C)(D) H^{*} d \log (A) B \\
& =\operatorname{Tr} H^{*}(d \log (A) B) H d \exp (C) D \\
& =\operatorname{Tr} d\left(H^{*}(\log A) H\right) B d \exp (C) D \\
& =\operatorname{Tr}(d(C) B) d \exp (C) D \\
& =\operatorname{Tr} D d \exp (C) D,
\end{aligned}
$$

we obtain

$$
d^{2} \varphi(A)(B, B) \leq Q(E, G)-\operatorname{Tr} D d \exp (C) D .
$$

We now apply Proposition 2.2 and obtain

$$
\begin{aligned}
Q(E, G) & =Q\left(H \exp (C) H^{*}, G\right) \\
& \leq Q\left(\exp C, H^{-1} G\left(H^{*}\right)^{-1}\right) \\
& =Q(\exp C, d \exp (C)(D)) \\
& =\operatorname{Tr} d \exp (C)(D) d \log (\exp C) d \exp (C)(D) .
\end{aligned}
$$


However, since the inverse of the linear map $h \rightarrow d \exp (x) h$ is given by

$$
d \exp (x)^{-1}=d \log (\exp x)
$$

we realise that

$$
d \log (\exp C) d \exp (C)(D)=d \exp (C)^{-1}(d \exp (C) D)=D .
$$

Therefore,

$$
Q(E, G) \leq \operatorname{Tr} D d \exp (C) D
$$

and thus $d^{2} \varphi(A)(B, B) \leq 0$ for positive definite $A$ and self-adjoint $B$. This shows that $\varphi$ is concave. QED

Corollary 3.2. Consider $n \times m$ matrices $H_{1}, \ldots, H_{k}$ with

$$
H_{1}^{*} H_{1}+\cdots+H_{k}^{*} H_{k} \leq 1_{n}
$$

where $1_{n}$ denotes the $n \times n$ unit matrix. Then the trace function

$$
\varphi\left(A_{1}, \ldots, A_{k}\right)=\operatorname{Tr} \exp \left(H_{1}^{*}\left(\log A_{1}\right) H_{1}+\cdots+H_{k}^{*}\left(\log A_{k}\right) H_{k}\right)
$$

is concave in $k$-tuples of positive definite $n \times n$ matrices.

Proof. We set

$$
A=\left(\begin{array}{cccc}
A_{1} & 0 & \cdots & 0 \\
0 & A_{2} & & 0 \\
\vdots & & \ddots & \vdots \\
0 & 0 & \ldots & A_{k}
\end{array}\right) \quad \text { and } H=\left(\begin{array}{cccc}
H_{1} & 0 & \cdots & 0 \\
H_{2} & 0 & \cdots & 0 \\
\vdots & \vdots & \ddots & \vdots \\
H_{k} & 0 & \cdots & 0
\end{array}\right)
$$

with zero matrices of suitable orders inserted and notice that $H$ is a contraction. Furthermore,

$$
H^{*}(\log A) H=\left(\begin{array}{cccc}
\sum_{i=1}^{k} H_{i}^{*}\left(\log A_{i}\right) H_{i} & 0 & \ldots & 0 \\
0 & 0 & \ldots & 0 \\
\vdots & \vdots & \ddots & \vdots \\
0 & 0 & \ldots & 0
\end{array}\right) .
$$

Thus

$$
\operatorname{Tr} \exp \left(H^{*}(\log A) H\right)=\operatorname{Tr} \exp \left(\sum_{i=1}^{k} H_{i}^{*}\left(\log A_{i}\right) H_{i}\right)+(k-1) n
$$

and the statement follows from Theorem 3.1. QED 
Corollary 3.3. Consider $n \times m$ matrices $H_{1}, \ldots, H_{k}$ with

$$
H_{1}^{*} H_{1}+\cdots+H_{k}^{*} H_{k} \leq 1_{n}
$$

and a self-adjoint $n \times n$ matrix $L$. Then the trace function

$$
\varphi\left(A_{1}, \ldots, A_{k}\right)=\operatorname{Tr} \exp \left(L+\sum_{i=1}^{k} H_{i}^{*}\left(\log A_{i}\right) H_{i}\right)
$$

is concave in $k$-tuples of positive definite $n \times n$ matrices.

Proof. By appealing to continuity we may without loss of generality assume

$$
H_{1}^{*} H_{1}+\cdots+H_{k}^{*} H_{k}<1_{n}
$$

and set $H_{k+1}=\left(1_{n}-\left(H_{1}^{*} H_{1}+\cdots+H_{k}^{*} H_{k}\right)\right)^{1 / 2}$. Then $H_{k+1}$ is positive definite and since

$$
H_{1}^{*} H_{1}+\cdots+H_{k}^{*} H_{k}+H_{k+1}^{2}=1_{n}
$$

we deduce from Corollary 3.2 that the trace function

$$
\begin{aligned}
& \varphi\left(A_{1}, \ldots, A_{k}, A_{k+1}\right) \\
& =\operatorname{Tr} \exp \left(H_{1}^{*}\left(\log A_{1}\right) H_{1}+\cdots+H_{k}^{*}\left(\log A_{k}\right) H_{k}+H_{k+1}\left(\log A_{k+1}\right) H_{k+1}\right)
\end{aligned}
$$

is concave in positive definite matrices. We keep $A_{k+1}$ constant by setting

$$
A_{k+1}=\exp \left(H_{k+1}^{-1} L H_{k+1}^{-1}\right)
$$

and the statement now follows. QED

Remark 3.4. Corollary 3.3 contains two celebrated theorems of Lieb. If we set $k=1$ and $H=1$ then the trace function

$$
\varphi(A)=\operatorname{Tr} \exp (L+\log A)
$$

is concave in positive definite matrices, cf. [7, Theorem 6]. If $H_{1}, \ldots, H_{k}$ are chosen as square roots of positive numbers times the identity matrix then we obtain that the trace function

$$
\varphi\left(A_{1}, \ldots, A_{k}\right)=\operatorname{Tr} \exp \left(L+p_{1} \log A_{1}+\cdots+p_{k} \log A_{k}\right),
$$

defined in positive definite matrices, is concave, where $p_{1}, \ldots, p_{k}$ are nonnegative numbers with $p_{1}+\cdots+p_{k} \leq 1$, cf. [7, Corollary 6.1 (1)]. 
Corollary 3.5. Let $L$ be a fixed self-adjoint matrix, and let $A_{1}, \ldots, A_{k}$ be random self-adjoint matrices. Then the inequality

$$
\mathbb{E} \operatorname{Tr} \exp \left(L+\sum_{i=1}^{k} H_{i}^{*} A_{i} H_{i}\right) \leq \operatorname{Tr} \exp \left(L+\sum_{i=1}^{k} H_{i}^{*}\left(\log \mathbb{E} e^{A_{i}}\right) H_{i}\right)
$$

holds for fixed matrices $H_{1}, \ldots, H_{k}$ with $H_{1}^{*} H_{1}+\cdots+H_{k}^{*} H_{k} \leq 1$.

The result follows directly from Corollary 3.3 by applying Jensen's inequality, cf. also [9, Corollary 3.3]. A simple consequence is that

$$
\mathbb{E} \operatorname{Tr} \exp \left(L+\frac{A_{1}+\cdots+A_{k}}{k}\right) \leq \operatorname{Tr} \exp \left(L+\frac{\log \mathbb{E} e^{A_{1}}+\cdots+\log \mathbb{E} e^{A_{k}}}{k}\right)
$$

for a fixed self-adjoint matrix $L$ and random self-adjoint matrices $A_{1}, \ldots, A_{k}$.

\section{Multivariate trace inequalities}

Lemma 4.1. Consider $n \times m$ matrices $H_{1}, \ldots, H_{k}$ with

$$
H_{1}^{*} H_{1}+\cdots+H_{k}^{*} H_{k}=1_{n}
$$

and a self-adjoint $n \times n$ matrix $L$. Then we have the inequality

$$
\begin{aligned}
& \operatorname{Tr} \exp \left(L+\sum_{j=1}^{k} H_{j}^{*}\left(\log B_{j}\right) H_{j}\right) \\
& \leq \operatorname{Tr} \exp \left(L+\sum_{j=1}^{k} H_{j}^{*}\left(\log A_{j}\right) H_{j}\right) \sum_{i=1}^{k} H_{i}^{*}\left(d \log \left(A_{i}\right) B_{i}\right) H_{i}
\end{aligned}
$$

for positive definite $n \times n$ matrices $A_{1}, \ldots, A_{k}$ and $B_{1}, \ldots, B_{k}$.

Proof. Since the trace function

$$
\varphi\left(A_{1}, \ldots, A_{k}\right)=\operatorname{Tr} \exp \left(L+\sum_{j=1}^{k} H_{j}^{*}\left(\log A_{j}\right) H_{j}\right)
$$

is concave and (positively) homogeneous, we may apply Lemma 2.1 and obtain the inequality

$$
d \varphi\left(A_{1}, \ldots, A_{k}\right)\left(B_{1}, \ldots, B_{k}\right) \geq \varphi\left(B_{1}, \ldots, B_{k}\right) .
$$


By applying the chain rule for Fréchet differentials we then derive

$$
\begin{aligned}
& \varphi\left(B_{1}, \ldots, B_{k}\right) \leq \sum_{i=1}^{k} d_{i} \varphi\left(A_{1}, \ldots, A_{k}\right) B_{i} \\
& =\sum_{i=1}^{k} \operatorname{Tr} d \exp \left(L+\sum_{j=1}^{k} H_{j}^{*}\left(\log A_{j}\right) H_{j}\right) H_{i}^{*}\left(d \log \left(A_{i}\right) B_{i}\right) H_{i} \\
& =\sum_{i=1}^{k} \operatorname{Tr} \exp \left(L+\sum_{j=1}^{k} H_{j}^{*}\left(\log A_{j}\right) H_{j}\right) H_{i}^{*}\left(d \log \left(A_{i}\right) B_{i}\right) H_{i}
\end{aligned}
$$

and the statement follows. QED

Theorem 4.2. Consider $n \times m$ matrices $H_{1}, \ldots, H_{k}$ with

$$
H_{1}^{*} H_{1}+\cdots+H_{k}^{*} H_{k}=1_{n} .
$$

Then we have the inequality

$$
\operatorname{Tr} \exp \left(L+\sum_{i=1}^{k} H_{i}^{*} B_{i} H_{i}\right) \leq \operatorname{Tr} \exp (L) \sum_{i=1}^{k} H_{i}^{*}\left(\exp B_{i}\right) H_{i}
$$

for arbitrary self-adjoint $n \times n$ matrices $L$ and $B_{1}, \ldots, B_{k}$.

Proof. Choose in Lemma 4.1 for $i=1, \ldots, k$ the matrix $A_{i}$ as the identity matrix. Then the Fréchet differential $d \log \left(A_{i}\right) B_{i}=B_{i}$ and $\log A_{i}=0$. The result then follows by replacing $B_{i}$ with $\exp B_{i}$ for $i=1, \ldots, k$. QED

The above inequality is a direct generalisation of the Golden-Thompson inequality. Indeed, if we put $k=1$ and take $H_{1}$ as the identity matrix then the inequality in Theorem 4.2 takes the form

$$
\operatorname{Tr} e^{L+B} \leq \operatorname{Tr} e^{L} e^{B},
$$

cf. [4, 8, 7]. We may obtain other corollaries of Lemma 4.1.

Theorem 4.3. Consider $n \times m$ matrices $H_{1}, \ldots, H_{k}$ with

$$
H_{1}^{*} H_{1}+\cdots+H_{k}^{*} H_{k}=1_{n} .
$$

Then we have the inequality

$$
\operatorname{Tr} \exp \left(\sum_{i=1}^{k} H_{i}^{*}\left(\log B_{i}-\log A_{i}\right) H_{i}\right) \leq \sum_{i=1}^{k} \operatorname{Tr} H_{i}^{*}\left(d \log \left(A_{i}\right) B_{i}\right) H_{i}
$$

for positive definite $n \times n$ matrices $A_{1}, \ldots, A_{k}$ and $B_{1}, \ldots, B_{k}$. 
Proof. The result follows by setting

$$
L=-\left(H_{1}^{*}\left(\log A_{1}\right) H_{1}+\cdots+H_{k}^{*}\left(\log A_{k}\right) H_{k}\right)
$$

in Lemma 4.1. QED

If we in Theorem 4.3 put $k=1$ and take $H_{1}$ as the unit matrix we obtain

$$
\operatorname{Tr} \exp (\log B-\log A) \leq \operatorname{Tr} d \log (A) B=\operatorname{Tr} A^{-1} B
$$

which is the Golden-Thompson inequality.

Furthermore, if $A_{i}$ and $B_{i}$ commute for $i=1, \ldots, k$ then Theorem 4.3 reduces to the inequality

$$
\operatorname{Tr} \exp \left(\sum_{i=1}^{k} H_{i}^{*}\left(\log B_{i}-\log A_{i}\right) H_{i}\right) \leq \sum_{i=1}^{k} \operatorname{Tr} H_{i}^{*} B_{i} A_{i}^{-1} H_{i}
$$

which is an expression of operator convexity of the exponential function under the trace.

Theorem 4.4. Consider $n \times m$ matrices $H_{1}, \ldots, H_{k}$ with

$$
H_{1}^{*} H_{1}+\cdots+H_{k}^{*} H_{k}=1_{n} .
$$

Then we have the inequality

$$
\begin{aligned}
& \operatorname{Tr} \exp \left(\sum_{i=1}^{k} H_{i}^{*}\left(\log B_{i}+\log C_{i}-\log A_{i}\right) H_{i}\right) \\
& \leq \operatorname{Tr} \exp \left(\sum_{i=1}^{k} H_{i}^{*}\left(\log C_{i}\right) H_{i}\right) \sum_{i=1}^{k} H_{i}^{*}\left(d \log \left(A_{i}\right) B_{i}\right) H_{i}
\end{aligned}
$$

for positive definite $n \times n$ matrices $A_{1}, \ldots, A_{k}, B_{1}, \ldots, B_{k}$ and $C_{1}, \ldots, C_{k}$.

Proof. The result follows by setting

$$
L=H_{1}^{*}\left(\log C_{1}-\log A_{1}\right) H_{1}+\cdots+H_{k}^{*}\left(\log C_{k}-\log A_{k}\right) H_{k}
$$

in Lemma 4.1. QED

If we in Theorem 4.4 put $k=1$ and take $H_{1}$ as the unit matrix we obtain

$$
\operatorname{Tr} \exp (\log B+\log C-\log A) \leq \operatorname{Tr} C d \log (A) B,
$$

which is the extended Golden-Thompson inequality. The extended GoldenThompson inequality reduces to the Golden-Thompson inequality if $A$ and $B$ commute. 


\section{References}

[1] T. Ando and F. Hiai. Log majorization and complementary Golden-Thompson type inequalities. Linear Algebra Appl., 197 (198):113-131, 1994.

[2] T.M. Flett. Differential Analysis. Cambridge University Press, Cambridge, 1980.

[3] P.J. Forrester and C.J. Thompson. The Golden-Thompson inequality: Historical aspects and random matrix applications. J. Math. Phys., 55(023503), 2014.

[4] S. Golden. Lower bounds for the Helmhotz function. Phys. Rev. B, 137(11271128), 1965.

[5] F. Hansen and Z. Zhang. Characterisation of matrix entropies. arXiv: 1402.2118, pages 1-11, 2014.

[6] F. Hiai and D. Petz. The Golden-Thomson trace inequality is complemented. Linear Algebra Appl., 181:153-185, 1993.

[7] E. Lieb. Convex trace functions and the Wigner-Yanase-Dyson conjecture. Advances in Math., 11:267-288, 1973.

[8] C.J. Thompson. Inequality with applications in statistical mechanics. J. Math. Phys., 6:1812-1813, 1965.

[9] J.A. Tropp. User-friendly tail bounds for sums of random variables. Found Comput Math, 12:389-434, 2012.

Frank Hansen: Institute for Excellence in Higher Education, Tohoku University, Japan.

Email: frank.hansen@m.tohoku.ac.jp. 